\title{
Reinventar la escuela: alternativas radicales exitosas del sur global*
}

\author{
Reinventing school: successfull radical \\ alternatives to the global south
}

Joseph P. Farrell, † Caroline Manion y Santiago Rincón-Gallardo**

\section{RESUMEN}

Artículo basado en el análisis de más de 200 programas educativos instalados en escuelas comunitarias en Colombia, México y Bangladesh, a cargo de un equipo internacional de investigadores que desarrollan la teoría "de la base hacia arriba" (grounded theory), aún en ciernes, en este ejercicio de educación comparada. La primera parte presenta algunas de las distinciones clave entre las formas de escolaridad formal y el modelo alternativo emergente. La siguiente ofrece un análisis comparado a detalle de tres casos ejemplares del modelo alternativo. El foco estará puesto en dos aspectos clave: el pedagógico y la formación docente. Al abordar los principales cuestionamientos a la escuela tradicional, se destacan algunas de las principales lecciones aprendidas hasta ahora sobre las formas alternativas. En la sección final, se plantean dos preguntas fundamentales, tanto empíricas como teóricas, cuyas respuestas quedan pendientes.

Palabras clave: escolarización formal, Escuela Nueva, escuela comunitaria, educación rural, educación comparada

\begin{abstract}
Article based on analysis of more than 200 educational programs installed in community schools in Colombia, Mexico and Bangladesh, in charge of an international team of researchers who developed the theory "from the bottom up" (grounded theory), still in the making, in this exercise of comparative education. The first part presents some of the key distinctions between formal schooling and the emerging alternative model. The following provides an analysis compared to three exemplary cases of the alternative model. The focus will be on two key issues: the pedagogical and teacher training. Addressing the main challenges to the traditional school, highlights some of the main lessons learned so far on alternative forms. In the final section, there are two fundamental questions, both empirical and theoretical, questions whose answers are pending.
\end{abstract}

Key words: formal schooling, new school, community school, rural education, comparative education

\footnotetext{
*Adaptación al español de: Farrell, P., Manion, C., y Rincón-Gallardo, S. "Reinventing Schooling: Successful Radical Alternatives from the Global South", en K. Bickmore, R. Hayhoe, C. Manion, K. Mundy y R. Read (Eds.). Comparative and International Education: Issues for Teachers, 2a ed., Capítulo 3. Toronto/Vancouver, Canadian Scholars, 2017, pp. 59-87. Traducción de S. Rincón Gallardo y publicado aquí con la autorización de los autores y de Canadian Scholars.

"* Ontario Institue for Studies in Education, University of Toronto; carly.manion@utoronto.ca; santiago.rincon.gallardo@utoronto.ca
} 


\section{INTRODUCCIÓN}

La adopción de ideas educativas ha sido una línea de trabajo importante de la educación comparada e internacional. Algunas formas de escolarización formal, concebidas originalmente en la Europa oriental a inicios del siglo XIX, se han diseminado a lo largo del mundo. A pesar de las grandes diferencias culturales, algunos aspectos clave de estas formas se han vuelto prácticamente universales. Desafortunadamente, una vez instaladas, han demostrado ser extremadamente difíciles de cambiar, al menos en gran escala. Mientras tanto, a partir de finales de los setenta, y ganando impulso desde los noventa, se ha gestado una revolución escolar silenciosa en el sur global que, en muchos casos, ha transformado dichas formas, especialmente en la educación primaria, aunque en algunos casos también en la educación secundaria.

Estas alternativas radicales, en muchos sentidos exitosas, se conocen poco y solo ocasionalmente reciben mención por parte de educadores e investigadores de la educación en el llamado primer mundo; por lo regular, no se les conoce o no se les entiende bien en sus países de origen. Ha comenzado a formarse un pequeño cuerpo de publicaciones sobre estas alternativas, pero consta, principalmente, de estudios de caso individuales con pocas referencias a otras prácticas, o de análisis comparados de conjuntos pequeños de algunos casos, generalmente de la misma región geopolítica (Anderson, 2002; Reimers, 2000).

Algunos de estos programas son todavía de menor escala, nuevos (por lo regular, en etapa de piloto), y no están muy bien documentados. Mucha de la información sobre ellos consiste en conocimiento que se distribuye de manera informal entre practicantes e investigadores. Otros, cuentan con varias décadas de experiencia, se han extendido a sistemas de miles (a veces decenas de miles) de escuelas, y disponen de sólida investigación y de evaluaciones de sus resultados. Algunos operan dentro de los marcos administrativos estándar de su Ministerio de Educación; otros son aplicados, por completo, por organizaciones no gubernamentales (ONG), mientras que otros tantos consisten en modelos mixtos con varias combinaciones de formas de planeación y gestión de gobiernos, ONG y de la sociedad civil. En casi todos, 
las escuelas participantes pueden considerarse dentro de la categoría de "educación comunitaria" o "escuelas comunitarias", con fuertes vínculos orgánicos entre las comunidades donde habitan los educandos. Estos vínculos adquieren diversas formas en distintos lugares, de acuerdo con la historia y los patrones locales de organización social; sea como fuere, son lazos fuertes y resultan cruciales. En la mayoría de las experiencias que han sido evaluadas, los resultados de estas escuelas son muy buenos en lo que concierne a matrícula, retención, eficiencia terminal, transición al siguiente nivel de escolaridad y logro educativo medible. Por lo regular, sus estudiantes obtienen puntajes en pruebas estándar que son, al menos, similares a, y con frecuencia mejores que aquellos de sus pares en escuelas regulares, incluso de niños y jóvenes en condiciones de mucho mayor privilegio. Las tasas de eficiencia terminal en primaria suelen estar por encima de $90 \%$, y la gran mayoría de quienes completan la primaria, transita hacia el nivel postprimario, por lo general, con resultados excelentes, incluso cuando la población objetivo ha sido la escuela secundaria, donde se observan tendencias similares: altos porcentajes de estudiantes que completan este nivel educativo y continúan a la preparatoria. Estos resultados son espectaculares, si se toma en cuenta que los nińos y jóvenes que acuden a estas escuelas se encuentran entre los más marginados del mundo y de las sociedades a las que pertenecen -es decir, los "más difíciles de alcanzar y los más difíciles de enseñar" en escuelas tradicionales.

Este artículo se basa en un análisis comparado en marcha de más de 200 de estos programas; ha estado a cargo de un equipo internacional de investigadores, investigadores de posgrado, líderes de agencias internacionales, diseñadores de programas, y codirigido por el primer autor. Como análisis comparado en desarrollo, se trata de un ejercicio internacional y comparado para el desarrollo de la teoría "de la base hacia arriba" (grounded theory), que está todavía en una etapa temprana. Hay mucho que aún no entendemos por completo y, con frecuencia, continúan surgiendo nuevas interrogantes. Es posible, sin embargo, distinguir con suficiente claridad algunos patrones y conclusiones que permiten escribir sobre ellos, incluso en esta etapa germinal. La primera sección de este trabajo se sustenta en una base de datos general 
para presentar algunas de las distinciones clave entre las formas de escolaridad formal y el modelo alternativo emergente (llamaremos a estas formas "malas noticias" y "buenas noticias", respectivamente). La siguiente sección ofrece un análisis comparado a detalle de tres casos ejemplares que han existido lo suficiente como para acumular una cantidad significativa de experiencia y evaluación y que, colectivamente, representan algunos de los principales patrones distintivos del modelo alternativo. Existe un rango amplio de preguntas y asuntos abordados en el programa de investigación comparada que está examinando estos modelos alternativos, pero el foco de este artículo estará puesto en dos aspectos clave. Primero, el aspecto pedagógico: ¿cómo es que los niños y jóvenes en estos programas alternativos aprenden tan bien y cómo logran hacerlo? ¿Qué es lo que sucede en estas aulas que produce estos resultados de aprendizaje? El segundo concierne a la formación docente: ¿cómo es que los maestros/facilitadores de estos programas aprenden rápidamente a ejecutar formas radicalmente distintas de la escolaridad formal y hacerlo bien? Al abordar estas preguntas, destacaremos algunas de las principales lecciones aprendidas hasta ahora. En la sección final, destacamos dos preguntas fundamentales, tanto empíricas como teóricas, $\mathrm{cu}-$ yas respuestas quedan pendientes.

El principal argumento aquí es que la mejor esperanza que tenemos para superar las malas noticias (como se describe más abajo) y, eventualmente, ofrecer mejores formas de aprender para esta y las futuras generaciones de jóvenes, en gran escala y tanto en naciones pobres como en países ricos es tratar de aprender de esta gente, aparentemente pequeña en número cuando se mira un lugar específico pero, de hecho, bastante grande en el agregado internacional y por lo regular en sitios con altos niveles de pobreza, que han logrado crear islas de éxito donde muchos otros han fracasado o solo han tenido éxito marginal. Un posible subtítulo para este artículo podría ser "Aprender del éxito". Si elegimos hacerlo, quizá ayudemos a revertir la tendencia de las dos últimas décadas, en que las ideas y los patrones educativos del Norte rico se han exportado o impuesto con tanta frecuencia en los dos tercios restantes de la población, los países pobres de nuestro mundo. 


\section{LAS MALAS Y LAS BUENAS NOTICIAS: LA ESCOLARIZACIÓN FORMAL}

Como educadores, somos a la vez observadores y partícipes de un patrón muy peculiar. A lo largo del último siglo o más, hemos aprendido mucho sobre cómo aprenden mejor los humanos, jóvenes y viejos (Bransford, Brown y Cocking, 2000; Olson, 2003). No obstante, nada de este conocimiento nuevo ha penetrado en las prácticas estándar de las escuelas formales donde, por lo general, continúan repitiéndose los rituales, tradiciones y concepciones de cómo ocurre el aprendizaje y qué es lo que más vale la pena aprender, desarrolladas hace más de un siglo primero en la Europa oriental y después diseminadas alrededor del mundo. Esta idea se ha repetido por muchos años, y nos somos los primeros ni los últimos en expresarlo (Abbott y Ryan, 2001; Caillods, 1989; Caine y Caine, 1997; Davies, 1995; Fagerlind y Sjostedt, 1990; Farrell, 1989, 1998, 2004, 2008; Polyzoi, Fullan y Anchan, 2003). A finales de los noventa se publicaron tres libros fundamentales que relataban e intentaban entender un siglo de intentos fallidos de reforma educativa en los Estados Unidos (Farrell, 2000; Ravitsch y Vinovskis, 1995; Tyack y Cuban, 1995). Las historias de escolarización formal disfuncional e iniciativas fallidas de reforma que se relatan en estos libros indicaban patrones que son generalizables a la cultura escolar alrededor del mundo. Una lección general es que el cambio educativo planeado es un proyecto mucho más difícil y cargado de riesgo que lo que se había pensado en los cincuenta y sesenta (Farrell, 1997). En las últimas tres décadas se han hecho intentos de cambiar sistemas educativos enteros alrededor del mundo. La base de conocimiento sobre "reforma educativa sistémica" ha crecido sustancialmente con el estudio comparado de sistemas escolares exitosos en el orbe, con publicaciones como el reporte de McKinsey "Cómo continúan mejorando los sistemas que más han mejorado" (Mourshed, Chijioke y Barber, 2010). Aun cuando hay ahora mayor claridad sobre cómo mejorar la enseñanza y el aprendizaje en sistemas educativos enteros (ver por ejemplo Fullan y Quinn, 2015; Fullan y Rincón-Gallardo, 2016; Hargreaves y Shirley, 2012; Zavadski, 2009; Sahlberg, 2011), existen todavía 
muchos más ejemplos de fracaso, o éxito mínimo, que de éxito contundente y sostenible.

A grandes rasgos, las revisiones de experiencias de reforma a lo largo del mundo llegan a conclusiones similares: Las propuestas de reforma educativa pocas veces se ejecutan. Si llegan a hacerlo (ya por la vía legislativa, por medio de reglas, ya por programas experimentales), pocas veces se implementan bien y a gran escala o tienden a diluirse tras pocos años, mientras que el sistema vuelve lentamente a su estado normal. En los pocos casos en que se hace bien, a gran escala y de manera sostenible, hay muy poca evidencia del impacto a largo plazo y a escala, en la misión básica del proyecto que llamamos escuela: posibilitar y mejorar la capacidad de los nińos y jóvenes de aprender con profundidad. Lo que se sabe acerca el aprendizaje humano no tiene prácticamente nada que ver con el modo en que continúan operando las escuelas. Las formas de escolarización formal, establecidas en el siglo XIX, reflejan las concepciones erróneas sobre el aprendizaje humano de las élites intelectuales, políticas y económicas de aquella época, fundamentalmente distinta de la actual. Pero ahora que están tan bien establecidas, es muy difícil cambiarlas, al menos en gran escala (Ver Elmore, 1996). El cuadro 1 esboza algunos de los aspectos distintivos de la escolarización formal, yuxtapuestos con los modelos alternativos de educación que se presentan más adelante en este trabajo.

\section{CUADRO 1. Aspectos característicos de la Escolarización Formal vs. Modelos Alternativos Emergentes}

\begin{tabular}{|l|l|}
\hline \multicolumn{1}{|c|}{ Escolarización Formal } & \multicolumn{1}{c|}{ Modelos Alternativos Emergentes } \\
\hline Foco en la enseñanza & Foco en el aprendizaje \\
\hline Hablar como principal práctica para enseñar & $\begin{array}{l}\text { Escuchar como principal práctica para fomentar el } \\
\text { aprendizaje }\end{array}$ \\
\hline $\begin{array}{l}\text { Clases frontales e instrucción al grupo entero como forma } \\
\text { pedagógica dominante }\end{array}$ & $\begin{array}{l}\text { Tutoría entre pares, trabajo individual y en grupos } \\
\text { pequeños como forma pedagógica dominante }\end{array}$ \\
\hline $\begin{array}{l}\text { Respuestas cortas a preguntas simples y calificaciones en } \\
\text { exámenes como material principal para la evaluación }\end{array}$ & $\begin{array}{l}\text { Fuentes múltiples de evidencia producidas por los } \\
\text { estudiantes y colectadas por los maestros de manera } \\
\text { continua: trabajo escrito, pensamiento en voz alta, } \\
\text { demostraciones públicas de lo aprendido }\end{array}$ \\
\hline $\begin{array}{l}\text { Evaluación sumativa de los estudiantes como evento } \\
\text { discreto, repetido periódicamente }\end{array}$ & $\begin{array}{l}\text { Evaluación formativa realizada de manera continua, } \\
\text { acompañada por retroalimentación en tiempo real }\end{array}$ \\
\hline
\end{tabular}


REINVENTAR LA ESCUELA. ALTERNATIVAS RADICALES EXITOSAS DEL SUR GLOBAL

\begin{tabular}{|c|c|}
\hline Escolarización Formal & Modelos Alternativos Emergentes \\
\hline $\begin{array}{l}\text { Responsabilidad por el trabajo y la disciplina en el aula } \\
\text { concentrada en el maestro }\end{array}$ & $\begin{array}{l}\text { Responsabilidad por el trabajo y la disciplina en el aula } \\
\text { distribuida entre los estudiantes y los adultos }\end{array}$ \\
\hline $\begin{array}{l}\text { Estudiantes sentados en fila, en silencio la mayoría del } \\
\text { tiempo, mirando y escuchando al maestro }\end{array}$ & $\begin{array}{l}\text { Estudiantes moviéndose libremente dentro y fuera del } \\
\text { aula para acceder a recursos de aprendizaje (p. e., la } \\
\text { biblioteca escolar, computadoras, etc.) y para consultar } \\
\text { con sus compañeros o con los adultos según se requiera } \\
\text { en el momento }\end{array}$ \\
\hline $\begin{array}{l}\text { Expectativa de que el grupo cubra contenido } \\
\text { predeterminado, establecido en el currículo nacional, } \\
\text { estatal, o provincial }\end{array}$ & $\begin{array}{l}\text { Organización flexible del currículo para atender a los } \\
\text { intereses y/o al paso de los estudiantes. Combinación } \\
\text { de currículo nacional/estatal y currículo relevante } \\
\text { localmente }\end{array}$ \\
\hline El grupo entero avanza al mismo paso & Cada estudiante sigue su propio ritmo \\
\hline $\begin{array}{l}\text { Día escolar organizado en bloques preestablecidos de } \\
\text { tiempo para cada materia }\end{array}$ & $\begin{array}{l}\text { Día escolar con estructura flexible que se adapta a las } \\
\text { necesidades, intereses y ritmo de los estudiantes }\end{array}$ \\
\hline $\begin{array}{l}\text { Maestros y directores como fuente única de autoridad } \\
\text { sobre la enseñanza }\end{array}$ & $\begin{array}{l}\text { Combinación de maestros formales, educadores en } \\
\text { formación, y recursos comunitarios (padres de familia, } \\
\text { otros miembros de la comunidad) involucrados } \\
\text { intencionalmente en la gestión de la escuela y el } \\
\text { aprendizaje de los estudiantes }\end{array}$ \\
\hline $\begin{array}{l}\text { Gobernanza y gestión escolar exclusivamente en manos } \\
\text { del personal administrativo }\end{array}$ & $\begin{array}{l}\text { Participación activa de los estudiantes y la comunidad en } \\
\text { la gobernanza y gestión de la escuela }\end{array}$ \\
\hline Organización de los estudiantes en grupos por edad & Organización multigrado \\
\hline Separación clara entre escuela y comunidad & $\begin{array}{l}\text { Flujo libre de niños y adultos entre la escuela y la } \\
\text { comunidad }\end{array}$ \\
\hline $\begin{array}{l}\text { Formación docente consiste principalmente de eventos } \\
\text { aislados, clases frontales, discusión de teorías recientes, } \\
\text { estrategias pedagógicas, y gestión del aula }\end{array}$ & $\begin{array}{l}\text { Formación continua e intensiva a maestros, mentoría } \\
\text { y apoyo entre pares, con oportunidades múltiples para } \\
\text { vivir, observar, practicar y refinar la práctica pedagógica } \\
\text { que se espera utilicen en sus aulas }\end{array}$ \\
\hline
\end{tabular}

Al mirarlas en conjunto, las características de la escolarización formal que se enlistan en la columna izquierda del cuadro ilustran el grado en que estas se han convertido en el modo "natural" de pensar y actuar en la escuela. ¿Quién las pondría, seriamente, en tela de juicio? Un aspecto llamativo de prácticamente todas las reformas educativas, ya sea orientadas a cambiar sistemas educativos enteros o escuelas individuales, es que raramente -o nunca- ponen en cuestión el modelo básico y las formas de la escolarización formal. Por lo regular, buscan alterar o mejorar una parte o pequeños componentes, sin cuestionar el resto.

La existencia de estos tipos de escolarización formal y su aparente intratabilidad en los esfuerzos de reforma educativa continúa siendo fuente de gran frustración para muchos ciudadanos 
que buscan mejores formas organizadas de aprendizaje para sus niños, para los líderes de reformas educativas que, por lo regular, ven fracasar sus esfuerzos, y para los investigadores del aprendizaje, que ven los hallazgos que con tanto esfuerzo han realizado tocar infructuosamente la puerta de la escuela.

Dentro del primer grupo ha habido, en años recientes, especial aunque no exclusivamente en Estados Unidos, un movimiento pequeño pero creciente orientado hacia las escuelas alternativas y la educación en el hogar (homeschooling) (Gloeckner y Jones, 2013; Martin-Chang, Gould y Meuse, 2011; Ray, 2013). Estos esfuerzos han resultado, en algunos casos, en escuelas o programas alternativos locales. En 2005, se estimaba que existían más de 12000 escuelas o programas alternativos en los Estados Unidos, y que al menos un millón de padres en ese país habían optado por alguna forma de educación en el hogar (Bauman, 2005); para 2010, el número de estudiantes educados en el hogar se estimaba en dos millones (Ray, 2011, citado en Gloeckner y Jones, 2013: 310). Pero estos esfuerzos representan una retirada del sistema formal de una cantidad relativamente pequeña de personas (es decir, 1 o $2 \%$ de la población), y no han tenido efectos observables en el sistema educativo formal más amplio.

En 2003, David Olson, reconocido psicólogo cognitivo, publicó un grito desesperado desde la investigación psicológica:

Por un tiempo me ha impactado el hecho de que mientras que la comprensión psicológica del aprendizaje y el desarrollo de los niños ha avanzado mucho... su impacto en la escolarización como práctica institucional ha sido modesto, si no es que nulo. Junto con la mayoría de mis colegas, había asumido que si tan solo supiéramos más acerca de cómo funciona la mente, cómo se desarrolla el cerebro, cómo toman forma los intereses, de qué maneras difieren las personas entre sí y, fundamentalmente, cómo aprende la gente, la práctica educativa daría un enorme salto hacia delante. Pero a pesar de que el conocimiento al respecto ha aumentado, las escuelas han permanecido notablemente inafectadas (Olson, 2003: ix).

Las observaciones recién vertidas aquí no deben llevarnos a concluir que no hay muchas buenas escuelas ni muchos buenos maestros en el mundo. Uno los puede encontrar con frecuencia, 
no solamente en lugares de privilegio, sino también en barrios urbanos y pueblos pobres, y es como encontrarse con un bello retoño en medio de un paisaje desolado. El problema crucial es que, como Michael Fullan y muchos otros han observado (Fullan y Watson, 1999), a la par que somos muy buenos para detectar y caracterizar una escuela buena, no tenemos aún alguna idea seria acerca de cómo crear tales escuelas, al menos en grandes números, ni sobre cómo transformar grandes cantidades de escuelas tradicionales en sitios que coincidan mejor con lo que se sabe ahora sobre el aprendizaje humano. Estas son las malas noticias.

Es claro también que muchas de las diferencias en las características de la educación formal y la alternativa representadas en el cuadro 1 pueden conceptualizarse no como dicotomías, sino como un continuo. Las formas de enseñanza y aprendizaje varían mucho con base en modos locales de entender complejos y culturalmente diversos. Los programas exitosos pueden tener rasgos que se encuentren más cerca o más lejos de los aspectos que parecieran, en términos "puros", diferenciar los dos modelos fundamentales descritos aquí. Pero aun con esta comparación, más bien genérica entre modelos de escolarización formal y modelos alternativos emergentes, hay algunos puntos que es de suma importancia considerar.

En primer lugar, ninguna de las ideas detrás de estos programas alternativos son particularmente nuevas. Han estado presentes en la literatura sobre currículo, pedagogía, psicología educativa y filosofía de la educación por largo tiempo. Existen, por ejemplo, escuelas comunitarias en grandes ciudades de Estados Unidos y en otros países (Melaville, Biert y Blank, 2006). Se han implementado varias formas de organización multigrado y de progresiones continuas de aprendizaje, ya sea por necesidad o intencionalmente, en muchas partes del mundo (ver, por ejemplo, la política de escuelas primarias del Gobierno de Quebec desde 2000 -Gouvernement du Québec, Ministère de l'Éducation, 2000)-). Muchos maestros han implementado varios de los aspectos que se enlistan en la columna derecha del cuadro 1 (ver, por ejemplo, Rodríguez, 2015). Pero, como sugerimos anteriormente, estos intentos han consistido, principalmente, de ejemplos aislados. 
Lo más importante que estos programas alternativos nos enseñan, en su conjunto, es que el modelo tradicional puede cambiar en gran escala. Y esto puede lograrse más claramente en sitios históricamente marginados, con recursos limitados, y con resultados de aprendizaje sobresalientes. Estos programas demuestran que las pedagogías activas, centradas en el niño, con participación robusta de los padres y la comunidad en el aprendizaje de sus estudiantes, funcionan bien. Y donde estos modelos pedagógicos se ejecutan bien, incluso modestamente bien, están produciendo mejoras importantes de aprendizaje entre los jóvenes más pobres y desaventajados. Tomando en cuenta las malas noticias ya descritas, se trata este de un hallazgo de extrema importancia. Cómo lograr esto en un sitio específico, sea este rico o pobre, es siempre un desafío, y la solución apropiada diferirá de un lugar a otro, dependiendo de la historia y las tradiciones locales, así como de las condiciones socioculturales y político-económicas. No existen recetas de "talla única" (one-size-fits-all) que ofrecer aquí. Pero saber que se puede y que se ha hecho aun en lugares con altos niveles de pobreza constituye un recurso a nuestra disposición, y en nosotros está decidir si lo utilizamos o no.

\section{ANÁLISIS COMPARADO DE ESCUELAS ALTERNATIVAS EN COLOMBIA, MÉXICO Y BANGLADESH}

En esta sección presentamos, a detalle, un análisis comparado de tres casos seleccionados entre una base de datos mucho mayor: Escuela Nueva en Colombia, las Redes de Tutoría en México, y el Programa de Educación Primaria Informal de BRAC (Comité Bangladesí de Avance Rural, por sus siglas en inglés) en Bangladesh. En el caso de Escuela Nueva y BRAC, nos basaremos en estudios de caso extensos y detallados sobre estos programas que se produjeron en 2004 (estos se citan en las notas finales y se destacan en las sugerencias de lecturas adicionales al final de este artículo) y que han sido actualizados con investigación y datos más recientes. El caso de las Redes de Tutoría se basa en investigación reciente llevada a cabo por uno de los autores de este artículo. Los estudios de caso se diseńaron para ofrecer no solo hechos - por ejemplo, la historia, el contexto, los resultados medibles de aprendizaje, los costos y los programas de formación 
docente-, sino también un mosaico narrativo de lo que ocurre en el día a día de las escuelas participantes en estos programas.

Cada uno de los tres casos que aquí se presentan fue elegido por diversas razones: están bien documentados y han sido evaluados, incluyendo observaciones de los propios participantes, así como evaluaciones de organizaciones o individuos externos; ejemplifican diversas aproximaciones a pedagogías alternativas, con un núcleo transcultural común de comprensión del aprendizaje humano, y los autores conocen estos programas íntimamente y pueden, por lo tanto, ofrecer descripciones de sus actividades del día a día -que es, en parte, lo que este artículo busca hacer patente-. Tras presentar un poco del contexto nacional y cultural en que estos casos se desarrollaron, haremos una breve introducción a cada uno de ellos.

\section{CONTEXTO GENERAL}

Los tres países en cuestión se clasifican, por lo general, como en desarrollo o pobres, pero lo son de maneras distintas que es importante destacar. El cuadro 2 ofrece información básica sobre las tres naciones, contrastada con información sobre Canadá como punto de referencia.

CUADRO 2. Producto Interno Bruto per cápita en cuatro países

\begin{tabular}{|l|c|c|c|c|}
\hline & Bangladesh & Colombia & México & Canadá \\
\hline PIB per cápita & $\$ 3340$ & $\$ 12600$ & $\$ 16700$ & $\$ 43400$ \\
\hline $\begin{array}{l}\text { Proporción del ingreso para } \\
\text { el quintil más pobre }\end{array}$ & $8.9 \%$ & $3.3 \%$ & $4.9 \%$ & $7.1 \%$ \\
\cline { 2 - 5 } & $(2000-2004)$ & $(2010-2015)$ & $(2010-2015)$ & $(2000-2004)$ \\
\hline $\begin{array}{l}\text { Tasa de alfabetización de } \\
\text { adultos }\end{array}$ & $62 \%$ & $95 \%$ & $95 \%$ & $99 \%$ \\
\hline
\end{tabular}

Fuentes: *Montos en dólares estadounidenses para el periodo 2010-2014. World Bank, data.worldbank.org/ indicator/NY.GNP.PCAP.PP.CD/countries?display=default; ${ }^{* *}$ World Bank, data.worldbank.org/indicator/ SI.DST.FRST.20; ${ }^{* *}$ UNESCO Institute for Statistics, data.uis.unesco.org/Index.aspx?DataSetCode=EDULIT_ DS\&popupcustomise $=$ true\&lang $=$ en $\#$

En el cuadro, el Producto Interno Bruto (PIB) es un índice muy grueso de cuánta riqueza y recursos hay disponibles por persona en un país específico (las cifras se presentan en dólares 
internacionales actuales). La línea siguiente ofrece estimaciones gruesas de la proporción de tal riqueza nacional disponible y utilizable para el $20 \%$ más pobre y, como tal, funciona como indicador de la desigualdad en la distribución del ingreso en cada país.

Obviamente, en los tres países las familias pobres y sus niños son mucho más pobres que la gente en una categoría de ingreso similar en un país como Canadá, pero existen diferencias importantes. A Bangladesh se le considera, por lo general, como una de las naciones más pobres en el mundo, y ser pobre ahí es muy diferente de ser pobre en naciones como Colombia y México, considerados típicamente como naciones de ingreso medio-alto en el mundo. Esto no quiere decir que la pobreza en Colombia o México sea necesariamente una condición menos difícil o desesperada que la pobreza en Bangladesh, pero tampoco se le puede considerar igual. Debemos también destacar que los tres países cuentan con tradiciones centenarias de pensamiento e investigación, que han contribuido de forma importante a cómo entendemos el mundo y nuestro lugar en él.

Las tasas de alfabetización en el cuadro son informativas. Incluso en una nación tan pobre como Bangladesh, más de $60 \%$ de los adultos están alfabetizados, mientras que el porcentaje en Colombia y en México es cercano al 100\%. Así, aun en sitios extremadamente pobres, hay mucha gente, millones de hecho, altamente educada, que ha asistido a instituciones de educación superior, es heredera de tradiciones intelectuales y espirituales ancestrales y comparte espacio nacional y cultural con grandes cantidades de gente que no goza de estos privilegios.

Colombia y México son, al igual que la mayoría de Norteamérica, herederos de la sociedad europea. Al igual que los otros países en Latinoamérica, fueron colonizados por los españoles a principios del siglo XVI, con la consecuente destrucción de muchos de sus pueblos y culturas y, en relativamente poco tiempo, se convirtieron en sociedades mestizas. Hacia inicios del siglo XIX consiguieron su independencia de los imperios coloniales europeos. Esto sucedió poco tiempo después de que los Estados Unidos consiguiera la suya propia, y décadas antes de que $\mathrm{Ca}-$ nadá, como ahora la conocemos, hiciera lo mismo. Dada esta historia, Colombia y México son herederos de una larga tradición 
intelectual, cultural y religiosa (predominantemente católica-romana, o bien cristiana) europea -en este caso, de la región sur de Europa-. No obstante, en los dos siglos que siguieron a su independencia, se desarrolló en América Latina una tradición intelectual -que incluye ideas sobre la enseńanza y el aprendizajetan única y distinta de la de los colonizadores europeos, como lo son las tradiciones intelectuales y educativas de una sociedad de Norteamérica como lo es Canadá. Tras varios años de vivir y trabajar en Latinoamérica, incluyendo Colombia y México, los autores han comprendido que muchos de los términos y frases que se utilizan ahí para hablar de la enseńanza y el aprendizaje, así como para expresar cómo se desarrollan los niños son difíciles de traducir al inglés o al francés y, más en general, a los modos en que en Norteamérica se piensa en tales cosas.

Bangladesh es heredero de una de las tradiciones más antiguas de la historia humana de que se tiene registro. A la larga historia de pensamiento y tradiciones espirituales Confucianista, Hinduista y Budista, el Islam se impuso en el siglo XII por medio de una invasión colonial desde la región central de Asia y el Medio oriente, cuna del Islam. Unos siglos después, los británicos invadieron y colonizaron el subcontinente Indio e impusieron otra capa, ahora europea, de tradición de pensamiento y tradición intelectual y espiritual. Tras la Segunda Guerra Mundial, el Raj británico fue desmantelado, con el desplazamiento sangriento y violento de pueblos hindúes y Musulmanes que condujo al establecimiento de dos estados en la antigua colonia: India y $\mathrm{Pa}-$ kistán, del cual Bangladesh conformaba la sección oriente. Esta partición no duró mucho, y a finales de los sesenta, estalló una guerra que condujo a la formación de la nueva nación independiente de Bangladesh.

Los tres casos que se presentan a continuación son breves e introductorios. Los estudios de caso a los que se hace referencia en las secciones siguientes ofrecen información y narrativas mucho más detalladas. 


\section{ESCUELA NUEVA EN COLOMBIA}

De los programas discutidos aquí, este es quizá el más conocido internacionalmente. Comenzó en muy pequeña escala a finales de los setenta como modelo alternativo para escuelas primarias multigrado en comunidades rurales colombianas. Posteriormente, Escuela Nueva fue cuidadosamente cultivada y desarrollada a través de la experimentación constante y de aprendizaje a partir de la práctica. En los ochenta, el Ministerio de Educación de Colombia adoptó Escuela Nueva como estrategia nacional para la educación rural en el país, convirtiéndose en uno de los cinco pilares del plan nacional para erradicar la pobreza extrema. Hacia finales de los ochenta, Escuela Nueva había alcanzado 20000 de las 34000 escuelas rurales en Colombia, con grados diversos de fidelidad en la implementación. En 1989, el modelo fue reconocido por el Banco Mundial como una de las tres políticas públicas de reforma más exitosas de los países en desarrollo (ver Psacharopoulos, Rojas y Vélez, 1992; Colbert y Arboleda, 1990; Farrell, 2003; Schiefelbein, 1993; Siabato, 1997). De acuerdo con un estudio internacional comparado, hacia finales de los noventa, gracias a Escuela Nueva, Colombia era el único país en Latinoamérica donde, excluyendo a las megaciudades, los estudiantes de escuela rural obtenían niveles de logro mayores que sus contrapartes en escuelas urbanas (McGinn, 1998).

A inicios de los noventa, una política nacional de descentralización lanzada en el contexto de una nueva constitución descarriló el plan nacional centralizado para la educación rural en Colombia y, desde entonces, Escuela Nueva se disipó al interior del ministerio (Benveniste y McEwan, 2001). Los líderes de Escuela Nueva dejaron el ministerio y, con el apoyo de varios exministros de educación, crearon Fundación Escuela Nueva con la intención de mantener vivo el modelo en escuelas rurales, y sostener la calidad de la innovación en regiones selectas. El modelo, además, ha sido adaptado a nuevos contextos y poblaciones, tanto en Colombia como internacionalmente. Al menos 15 países en Latinoamérica, África y el Sudeste Asiático lo han adoptado, de modo que este ha alcanzado a más de cinco millones de estudiantes. En Colombia, el modelo ha sido adaptado para 
servir a regiones urbano marginales, organizaciones de mujeres, y poblaciones migrantes y desplazadas. En 2013, Vicky Colbert, cocreadora de Escuela Nueva y fundadora y directora de Fundación Escuela Nueva, recibió el premio de la cumbre mundial de innovación (WISE, por sus siglas en inglés) en reconocimiento al impacto positivo de Escuela Nueva en las vidas de millones de niños y jóvenes a lo largo del mundo (ver Pitt, 2002; 2004).

\section{LAS REDES DE TUTORÍA MEXICANAS}

Las redes de tutoría mexicanas (denominadas Proyecto Comunidades de Aprendizaje, o Learning Community Project en algunas publicaciones internacionales) comenzaron en 2003, como una iniciativa de cambio pedagógico de base a pequeńa escala, encabezada por una pequeña organización no gubernamental llamada entonces Convivencia Educativa, A. C. (Actualmente, Redes de Tutoría/Aprender con Interés). La intención de esta iniciativa era convertir salones de clase de escuelas secundarias rurales convencionales en comunidades de aprendizaje, mediante una pedagogía basada en relaciones de diálogo, tutoría, y aprendizaje recíproco entre adultos y jóvenes. Entre 2003 y 2008, las redes de tutoría se habían extendido a más de 300 escuelas a lo largo del país, a través de la movilización, la activación y creación de redes sociales por parte de maestros, autoridades locales, y líderes del proyecto. En 2009, el proyecto inspiró la creación del Programa para la Mejora del Logro Educativo (PEMLE), una estrategia nacional dirigida a transformar radicalmente la enseńanza y el aprendizaje en las nueve mil escuelas con más bajo logro académico en México. Entre 2009 y 2012, las escuelas en que operó PEMLE habían aumentado el porcentaje de estudiantes que calificaban en niveles bueno y excelente en las pruebas nacionales de matemáticas y lenguaje, a un paso más acelerado, y alcanzando o incluso rebasando, a escuelas que atienden a estudiantes en condiciones de mayor privilegio (ver Rincón-Gallardo, 2016).

En 2012, con el regreso del partido político que históricamente había gobernado México hasta el 2000, la nueva dirección de la Secretaría de Educación Pública (SEP) cambió prioridades y pidió al equipo nacional del PEMLE que dejara la Secretaría, po- 
niendo un fin abrupto al programa. Varios miembros de PEMLE se reorganizaron en una pequeña organización no gubernamental (Aprender con Interés/Redes de Tutoría), que actualmente continúa apoyando la creación y diseminación de redes de tutoría en escuelas, regiones, y estados. Más recientemente, las redes de tutoría han sido adoptadas como eje de transformación pedagógica en el Consejo Nacional de Fomento Educativo (Conafe), órgano descentralizado del gobierno federal que atiende a las comunidades más pequeñas y marginadas del país a través de 30 mil centros educativos. Asimismo, comienzan a promoverse en escuelas rurales y urbanas de Chile a través del trabajo de la organización Educación 2020 y en escuelas de Tailandia y Singapur. ${ }^{1}$ Las redes de tutoría han sido señaladas por prominentes líderes del cambio educativo en el mundo y como ejemplo de una nueva pedagogía para el aprendizaje en profundidad (Fullan y Langworthy, 2014), del futuro del aprendizaje (City, Elmore y Lynch, 2012) y de los nuevos imperativos morales del cambio educativo (Shirley, 2016).

\section{PROGRAMA DE EDUCACIÓN PRIMARIA NO-FORMAL DEL COMITÉ BANGLADESÍ PARA EL AVANCE RURAL (BRAC)}

Este programa es otro de los "abuelos" en el campo de la innovación pedagógica en el sur global. Iniciado a mediados de los ochenta, se ha expandido hasta involucrar a cerca de $37 \mathrm{mil}$ escuelas rurales en Bangladesh y, poco a poco, se ha ido introduciendo en escuelas urbanas y en regiones con minorías étnicas a lo largo del país, en parte, mediante un programa de difusión con otras organizaciones locales. De manera similar a Escuela Nueva, se ha adaptado y adoptado, con apoyo de varias fundaciones y agencias internacionales, en países como Etiopía, Sudán, Somalia, Uganda, Sierra Leona y Afganistán (Ahmed, 1993; Haiplik, 2004; Scott, 1996; Sweetser, 1999). Más de 900000 estudiantes a lo largo del mundo están inscritos en programas de BRAC (BRAC Education Programme, 2016).

\footnotetext{
${ }^{1}$ Estas dos oraciones han sido añadidas por Santiago Rincón-Gallardo en la versión en español que aquí se presenta, para actualizar la información disponible al momento de entregar el capítulo original en inglés a la casa editorial.
} 


\section{RESULTADOS, COSTOS Y CURRÍCULO}

No tendría mucho caso comparar y analizar estos programas y el conjunto más amplio de iniciativas que representan, a menos que se demuestre que están produciendo buenos resultados de aprendizaje, no solo para aquellos nińos y jóvenes que logran completar la escuela, sino para la mayoría o todos los involucrados. Y estos programas deben lograr hacerlo a un costo -tanto total como por estudiante- sostenible. Está bien establecido ya que entre la mayoría de los grupos más pobres en las naciones ricas, el logro académico suele ser más bajo, y las tasas de deserción más altas que lo que se desearía. Como se mencionó antes, los tres casos que presentamos han sido evaluados -en mayor medida en los casos Escuela Nueva y BRAC-. Los resultados se presentan y discuten en mayor detalle en los estudios de caso a los que se hizo referencia en las secciones anteriores. Baste, por ahora decir que en todos los casos, los niños y jóvenes pobres acuden a la escuela, permanecen en ella, completan el ciclo escolar, aprenden no solo el contenido y las destrezas necesarias, sino que también desarrollan confianza y autoestima, y muchos de ellos continúan estudiando con éxito en el nivel siguiente de su trayectoria educativa. Y todo esto es en niveles similares o superiores a los de sus compatriotas en escuelas tradicionales, quienes provienen de contextos sociales más aventajados. Más aún, cuando se han hecho estudios de costo-beneficio, los costos por graduado exitoso son menores que en las escuelas tradicionales (deStefano, Hartwell, Schun Moore y Benbow, 2005).

En lo que respecta al currículo, todos estos programas siguen el currículo estándar nacional, si este se entiende como el conjunto de metas y objetivos para un ciclo o etapa específicos de aprendizaje. Lo que estos programas logran, mediante la alteración fundamental del modelo pedagógico, es una forma de aprender el currículo en un grado, por lo general, superior que el de las escuelas tradicionales, en ocasiones en un periodo de tiempo considerablemente más corto. Además, ofrecen oportunidades para que los niños y jóvenes aprendan contenidos relevantes localmente, e incluyen en el aprendizaje aspectos tales como la educación democrática y ciudadana (Escuela Nueva es particu- 
larmente fuerte en esta área), educación artística (el programa de BRAC es especialmente fuerte aquí) o en destrezas pedagógicas como tutores de otros - tanto jóvenes como adultos (más evidente en el caso de las Redes de Tutoría mexicanas).

\section{PEDAGOGÍA}

El cuadro 1 ofrece un esbozo de las pedagogías que utilizan estos programas. Todos estos, de una u otra manera, se han alejado del modelo pedagógico tradicional, separado por grados según edad, homogéneo y encajonado. Este cambio permite y fomenta el aprendizaje progresivo y continuo (los estudiantes avanzan individualmente o en grupos pequeños a su propio ritmo, y a ritmos distintos en distintas áreas de aprendizaje -se asume que, por ejemplo, un niño de cualquier edad puede ser muy hábil para leer, pero no tanto para resolver problemas matemáticos) y la tutoría entre pares (chicos mayores o más avanzados asisten a sus colegas más jóvenes o menos avanzados)-. En los tres casos que aquí se presentan, los grupos de estudiantes son multigrado, con estudiantes de varias edades y grados, aprendiendo juntos en la misma aula. El programa de BRAC está organizado, de cierta manera, en grados según edad, pues los niños en cualquiera de las escuelas participantes pasan por el primero, segundo grado, etc., pero estos grados no están definidos por el calendario, sino por el juicio de los facilitadores respecto de cuándo está listo cada estudiante para pasar a la siguiente etapa en un área de aprendizaje específica. Así, un grupo podría estar trabajando, por ejemplo, en lectura de tercer grado, matemáticas de segundo grado, y ciencias en cuarto grado simultáneamente. El grupo está formado por niños de distintas edades, y avanza junto a lo largo del programa de educación primaria, hasta cubrir un currículo de cinco años en cuatro, con el o los mismos maestros de principio a fin. Así, el programa es un medio que se adapta localmente para lograr objetivos pedagógicos fundamentales. Escuela Nueva es enteramente multigrado en el caso de la educación primaria. Cuenta con mecanismos de promoción flexibles que permiten a los estudiantes avanzar a su propio ritmo. Es común en Escuela Nueva ver a estudiantes trabajando en equipos pequeńos, cada uno estudiando un tema distinto. 
El modelo de Escuela Nueva en secundaria está organizado por grados, y las clases se dividen por materia, lo que refleja la organización y las rutinas de la escuela secundaria convencional. Aun así, el modelo mantiene varios elementos de trabajo multigrado, multiedad, y de apoyo entre pares en su pedagogía. Las Redes de Tutoría se introdujeron inicialmente en escuelas secundarias multigrado y gradualmente se extendieron hacia escuelas organizadas por grado. La pedagogía fundamental de relaciones de tutoría, no obstante, puede adaptarse fácilmente a aulas multigrado u organizadas por grado. Los estudiantes eligen sus temas de estudio de un catálogo de temas que el maestro o alguien más en el grupo domina. A cada estudiante se le asigna un tutor ya sea un adulto u otro estudiante-, quien apoya el proceso de aprendizaje a través de diálogo y tutoría. Cada estudiante sigue líneas de estudio a su propio paso. El énfasis de las Redes de Tutoría está en desarrollar destrezas de aprendizaje independiente, de modo que cubrir el currículo entero no es tan importante como asegurar que los estudiantes mejoran su habilidad para aprender por cuenta propia con cada nuevo proyecto de investigación.

En los modelos que recién hemos descrito, los estudiantes, por lo regular, utilizan una buena parte del día escolar trabajando individualmente o en grupos pequeños, en "esquinas" o centros de aprendizaje, en algunos casos utilizando materiales diseñados específicamente para el aprendizaje autoguiado. Se utilizan libros de texto estándar también, pero en conjunto con estos materiales especiales. Como es de esperarse, los libros de texto estándar, diseñados para grupos de edad específicos, no funcionan bien como única fuente de información para una escuela multigrado y multiedad. Cuando un estudiante o un grupo pequeño encuentra una dificultad, por lo regular acuden primero a estudiantes mayores o más avanzados para solicitar apoyo. Si eso no resuelve la dificultad, entonces piden ayuda al maestro o facilitador. Los adultos pasan la mayoría del tiempo moviéndose al interior del aula, checando el progreso de cada estudiante o grupo pequeńo, ayudando a resolver dudas, y llevando registro del progreso y dificultades individuales y del grupo para planear el trabajo futuro.

Esto no necesariamente implica que los maestros no enseñan, sino que lo hacen de una forma distinta. Trabajan sobre todo con 
estudiantes individuales o en grupos pequeños, más que con el grupo entero, respondiendo a las necesidades de aprendizaje en el momento en que estas emergen. También concentran parte de su esfuerzo en enseñar a cada grupo de estudiantes nuevos a leer, utilizando una variedad de métodos de enseñanza que resultarían familiares a casi cualquier maestro de primaria en el mundo, hasta que estos han alcanzado un nivel de decodificación y comprensión de textos escritos que les permite trabajar con materiales de autoaprendizaje. Los maestros pueden concentrar sus esfuerzos en desafíos de esta naturaleza, precisamente porque cuentan con que la mayoría de los estudiantes están involucrados la mayor parte del tiempo en el aprendizaje por cuenta propia. Salones como estos son lugares donde se percibe mucha actividad y movimiento, por lo general ruidosos, no con el ruido disruptivo de niños rebelándose o queriendo llamar la atención, sino con el productivo de nińos y jóvenes que trabajan juntos en su propio aprendizaje.

La figura 1 compara estos tres programas en una variedad de aspectos pedagógicos que pueden entenderse mejor como categorías continuas, más que discretas. Dos de estos aspectos se relacionan con la organización del día y el año escolares: periodos predeterminados - por materia y tipo de actividad-, vs. el flujo libre del tiempo; ciclos diarios y anuales estándar vs. adaptaciones locales. El siguiente conjunto de continuos intenta ubicar estos programas en categorías, ahora estándar, de diferencias pedagógicas: centrado en el maestro vs. centrado en el alumno; aprendizaje pasivo vs. aprendizaje activo; y enseńanza frontal vs. enseńanza constructivista. Es importante aquí hacer una aclaración: la ubicación de cada uno de los casos en los continuos representa tan solo aproximaciones basadas en el juicio informado y las observaciones cuidadosas de estos programas -aunque se trata de observaciones de un número necesariamente pequeño de las escuelas que participan en estos programas. 
FIGURA 1. Algunos continuos pedagógicos

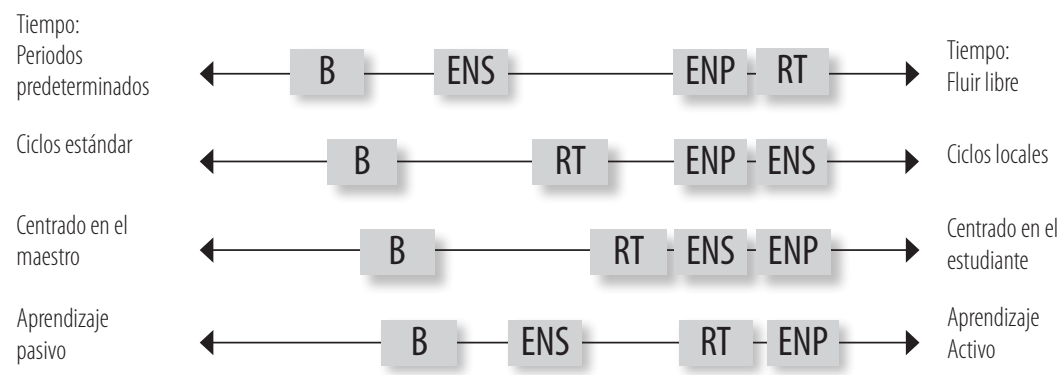

Códigos. ENP: Escuela Nueva Primaria; ENS: Escuela Nueva Secundaria; B: BRAC-Programa EPNF; RT: Redes de Tutoría

Más aún, en cualquier programa de escala modesta o grande, es de esperarse que haya variación, sea esta intencional o no, y estos programas no son la excepción. Así, la ubicación que damos a cada programa en los continuos no ha de considerarse como exacta, sino como nuestra mejor aproximación, con variación considerable alrededor de cada punto. Con esta aclaración en mente, la primera cosa que destacar es que Escuela Nueva en primarias y las Redes de Tutoría están en, o cerca del extremo derecho. Se trata de ejemplos que, implementados de lleno, ejemplifican de manera más completa la alternativa emergente. Pero es igualmente importante destacar que todos estos programas se han alejado considerablemente de las formas tradicionales de la escuela formal, caracterizadas en el lado izquierdo de la figura 1. Dado que todos estos han alcanzado niveles considerables de éxito en términos de mejor acceso, retención y, lo que es aún más importante, aprendizaje, podemos sugerir que diversos grados y combinaciones de alejamientos del modelo tradicional, adecuados a condiciones y tradiciones locales, pueden tener éxito. Como hemos enfatizado antes, no se espera ni se desea que el modelo emergente sea una receta homogénea para el éxito escolar, sino una herramienta para pensar en estos asuntos y aprender de la experiencia de otros. 


\section{MAESTROS: QUIÉNES SON Y CÓMO APRENDEN}

Es obvio que los maestros son la clave para cualquier sistema de mejora del aprendizaje. Por ello tiene sentido preguntarse: ¿quiénes son los maestros en estos programas alternativos? ¿De dónde vienen?, y ¿cómo aprenden esta forma radicalmente alternativa de trabajar con los chicos y las chicas en las escuelas? En nuestro repositorio de datos, pueden identificarse dos patrones distintos en términos de la selección de maestros. En el primer patrón, los maestros no se encuentran certificados formalmente según los sistemas estándar de selección y formación docente (como lo son las facultades de educación o las escuelas normales). Más bien, se trata de jóvenes, en su mayoría mujeres, con un grado modesto de educación escolar formal, por lo regular secundaria o preparatoria. Se trata de personas conocidas localmente, elegidas por un comité escolar local, y capacitadas en el área local para enseñar en la localidad. En gran medida son parte de la comunidad y conocidos por la comunidad. Se les llama con frecuencia facilitadores más que maestros, para evitar confusión y conflicto con las asociaciones o sindicatos de maestros. El programa bangladesí ejemplifica este patrón. Los ejemplos colombiano y mexicano representan el otro patrón común, en que los maestros tienen formación universitaria y están certificados (al igual que todos los maestros en el sistema educativo formal) y con frecuencia se desplazan de una escuela a otra en cumplimiento de reglas estándar concernientes a los maestros certificados en sus burocracias nacionales o regionales. Este ha sido un problema para Escuela Nueva y las Redes de Tutoría, pues las rutinas de la burocracia de la profesión docente y las regulaciones del ministerio o secretaría de educación crean con frecuencia un desplazamiento tal y tan frecuente de los maestros, que la continuidad del modelo alternativo puede complicarse, lo que crea la necesidad regular y constante de formar a los nuevos maestros en la esta modalidad.

Lo que llama la atención, no obstante, es que sin importar el mecanismo de selección de maestros en funciones, ya sea con facilitadores con no más de diez años de escolaridad, o con maestros con hasta 16 o más años de educación formal (es decir, con estudios universitarios o incluso posgrado), en todos estos casos 
los adultos aprenden rápidamente cómo trabajar en una forma pedagógica radicalmente distinta con excelentes resultados para sus estudiantes. En todos los casos, las actividades de formación docente inicial son breves (máximo unas pocas semanas) pero intensivas, e incluyen la observación de y la práctica de la pedagogía en escuelas modelo. A esto siguen varios años de programas bastante intensos de mentoría entre maestros y supervisión y apoyo regulares. En Escuela Nueva y las Redes de Tutoría, los maestros de una comunidad determinada son apoyados o exhortados a formar y participar en comunidades de práctica (llamadas microcentros en el caso de Escuela Nueva), donde se reúnen entre una y cuatro veces al mes para analizar problemas y discutir resultados. Hablar sobre los logros, compartir dudas, y pensar en voz alta ayuda a los maestros a avanzar hacia soluciones específicas, probarlas, y compartir los resultados de estas experiencias. Estas comunidades de práctica reducen la incertidumbre y el miedo que muchos maestros enfrentan al poner en marcha nuevas prácticas pedagógicas (Schiefelbein, 2006). Las Redes de Tutoría establecieron vías adicionales de consolidación de la nueva pedagogía de relaciones tutoras en salones de clase, incluyendo visitas mensuales por parte de un asesor que puede pasar entre uno y cinco días en la escuela, trabajando directamente con los maestros en las tardes para modelar la práctica de la tutoría, y en las mañanas para modelar y practicar junto con los maestros la nueva pedagogía con los estudiantes. La formación en servicio de los facilitadores que participan en el programa de BRAC es igualmente intensiva (Haiplik, 2004) y, de hecho, este es un aspecto característico de prácticamente todos los casos en nuestra base de datos más amplia.

Es importante destacar cuán distinto es este modo de formación y aprendizaje docente del que típicamente se encuentra en Norteamérica y en otras regiones desarrolladas, donde por lo regular se sobrecarga la parte inicial del proceso, con la mayoría de los recursos y energía para el desarrollo profesional puestos en el periodo previo al servicio. Una vez que los nuevos maestros han sido capacitados y contratados, prácticamente se les abandona a sus propios medios. Experiencias de aprendizaje profesional continuo son más bien poco frecuentes, esporádicas, y por lo regular 
diseñadas por niveles de autoridad muy alejados del aula. (Existen algunas pocas excepciones, pero el patrón general se mantiene).

Los programas que hemos considerado aquí demuestran que, contrario a la creencia popular a lo largo del mundo, los maestros no son obstáculo a cambios escolares fundamentales: no es necesario hacer reformas "a prueba de maestro", como parecen creer quienes promueven estándares y rendición de cuentas en la educación norteamericana y en varios países de Europa. Por el contrario, en estos casos, los maestros son los promotores y agentes de dicho cambio, aun cuando -y quizá precisamente porque - trabajan en condiciones muy difíciles, con frecuencia tienen una formación académica relativamente débil, y en ocasiones se les paga muy mal. Al igual que los niños y jóvenes en relativa "desventaja" a quienes sirven, pueden y de hecho logran resultados notables de aprendizaje y cambio en periodos muy cortos de tiempo. Existe un paralelo importante entre aprendices jóvenes y adultos aquí. Así como el éxito de los jóvenes parece basarse fundamentalmente en poner el foco en el aprendizaje más que en la enseñanza, pareciera que la transformación de los maestros se basa en el mismo cambio de foco. En estos sistemas el rol de los supervisores se ha modificado fundamentalmente. Más que vigilantes o administradores de reglas y procedimientos administrativos, son posibilitadores y promotores del aprendizaje de los maestros. Estos programas exitosos de cambio educativo, por lo general se diseminan o son llevados a escala no a través de reformas planeadas y dirigidas desde el centro con metas y objetivos definidos a la distancia, o con agentes o supervisores de una institución nacional o regional yendo a enseñar a los maestros sobre la idea o el marco conceptual más reciente, sino más bien a través de un proceso de innovación y difusión: maestros que aprenden de otros maestros, que comparten su "conocimiento profesional práctico" (Clandinin y Connelly, 1998) y sus destrezas docentes con otros maestros, y explorando juntos cómo su conocimiento y experiencia, compartidos y crecientes, pueden ayudar a todos, pero sobre todo, a los y las jóvenes y nińos a su cargo. 


\section{PREGUNTAS PENDIENTES}

En este esfuerzo de largo aliento aguardan muchas preguntas, perplejidades y desafíos. Enfatizaremos tan solo cuatro por ahora. El primero es que las publicaciones disponibles sobre estos programas son por lo general muy laudatorias, y esto podría dar la impresión de que se trata de parangones pedagógicos de la virtud. No lo son. Se trata, en todos los casos, de instituciones claramente humanas, por lo regular, mucho mejores que las escuelas tradicionales, pero con mucha variabilidad al interior de y entre los programas. Entre los muchos éxitos, se han cometido también errores -y en los mejores casos, estos han sido corregidos-. La gente involucrada en estos programas aprende en la marcha, pero lo importante es que aprenden. No se ha desarrollado aún, y es muy necesaria, literatura crítica que refleje de manera más completa tanto lo perfectible como el éxito de aquellos.

Un segundo punto concierne a la escala y la sustentabilidad. Tanto Escuela Nueva como las Redes de Tutoría fueron adoptadas, en algún momento de sus historias, como parte de estrategias nacionales para mejorar la educación rural, llegando a miles de escuelas (20 mil en el caso de Colombia, nueve mil en el caso de México), y mostrando un impacto positivo en el aprendizaje de los estudiantes en un tiempo relativamente corto. No obstante, ambas iniciativas fueron marginadas o expulsadas de la agenda nacional en sus países luego de que cambios del partido en el poder o de orientación de la política nacional definieron nuevas prioridades. Tanto Escuela Nueva como las Redes de Tutoría continúan vivas a través de organizaciones no gubernamentales creadas explícitamente con este propósito, pero la escala ha sido reducida de manera considerable. (Cabe destacar aquí que la adopción de las Redes de Tutoría como modelo pedagógico rector en el Consejo Nacional de Fomento Educativo en México está presentando una nueva oportunidad de extender el nuevo modelo pedagógico a las 30 mil comunidades más alejadas del país). En el caso de Bangladesh, a pesar de la transferencia de este modelo hacia otros países del sur Global, BRAC no ha podido hasta ahora alcanzar la escala necesaria para satisfacer las necesi- 
dades educativas de la mayoría de las comunidades rurales y otras comunidades históricamente marginadas.

Un tercer desafío tiene que ver con la fricción y tensión entre lo global y lo local. Todos estos casos se han nutrido de ideas y tradiciones educativas y pedagógicas que se conocen bien en el norte y occidente globales. La mayoría de la gente involucrada en estos modelos alternativos con quien hemos hablado y trabajado conoce bien la literatura estándar sobre educación y aprendizaje del norte y oriente globales. Han estudiado y conocen bien las ideas de iconos tales como John Dewey, María Montessori, y Johann Pestalozzi, así como nuevos pensadores como Howard Gardner, Nel Noddings, y muchos otros y, por lo general, están conscientes de las corrientes de pensamiento crítico occidental. Lo que han hecho es tomar estas ideas y perspectivas globalizadas y combinarlas con sus propias tradiciones y conocimiento sobre la enseńanza y el aprendizaje para desarrollar, poco a poco, modos sumamente efectivos y localmente apropiados para posibilitar el buen aprendizaje de los niños y niñas, especialmente los más pobres. Algo que ha llamado repetidamente nuestra atención cuando frecuentamos escuelas como estas, intentando entender cómo y por qué funcionan tan bien es que, en cierto modo, todas ellas se sienten bastante similares, aunque son tan distintas en otros sentidos. La Colombia rural no es el Bangladesh rural o el México rural. Pero existen similitudes que uno puede ver y sentir como educador con varios años de experiencia. Hacer sentido de esto, no como mero ejercicio teórico, sino a través de trabajo cuidadoso en el campo es un desafío de investigación de largo plazo, esencial si se quiere comenzar a entender en verdad la cuestión del vínculo entre lo global y lo local.

Hay un cuarto desafío quizá más intimidante, que tiene que ver con lo inadecuado que resultan muchos de nuestros términos y categorías estándar, derivados de la larga historia de las formas de la escolarización formal, para capturar con precisión lo que lentamente estamos comprendiendo sobre estos programas alternativos. Uno de estos aspectos es la distinción ahora común entre educación formal y no formal. Esta distinción se introdujo en la literatura especializada en los sesenta y setenta y fue importante entonces para denotar que la educación, como tarea institu- 
cional organizada, podía ser $y$, de hecho, era provista por varios medios más allá de la escuela formal, para la gente joven, pero particularmente para los adultos (Ahmed, 1975; Coombs, 1976; Coombs y Ahmed, 1974; Evans, 1981). Pero, ¿qué hacer con esta categorización cuando encontramos, como ahora, que muchas de estos programas alternativos están trayendo al interior de las aulas los modelos pedagógicos de la educación no formal, incluyendo métodos que por mucho tiempo fueron defendidos por los educadores de adultos? Así, nuestras cómodas distinciones entre formal y no formal o entre aprendizaje de los adultos y de los nińos parecen ser anuladas por quienes participan en estos programas. Al parecer, no contamos aún con un lenguaje práctico y teórico para representar esta realidad (Rogers, 2004). Esto aplica también a términos como pedagogía centrada-en-el-niño o enseńanza activa o constructivista. Al intentar ajustar estos y muchos otros y variados programas a los términos que utilizamos normalmente en nuestras discusiones sobre la pedagogía y la escuela, hemos encontrado que los términos y categorías estándar no son del todo adecuados a lo que observamos. Estos se basan en una perspectiva de la enseñanza y el aprendizaje arraigada en la larga historia y las suposiciones implícitas de la escuela formal como la hemos entendido. Romper con estas categorías y suposiciones intelectuales tradicionales será quizá aún más difícil que romper con las formas tradicionales de práctica en las escuelas tal como las hemos conocido. En conclusión, hay mucho que aprender, y estamos en momentos iniciales. Pero hay mucho disponible de lo cual podemos aprender, si así elegimos hacerlo.

\section{BIBLIOGRAFÍA}

Abbott, John, y Ryan, Terry. The unfinished revolution: Learning, human behavior, communities and political paradox. Alexandria, VA, Association for Supervision and Curriculum Development, 2001.

Ahmed, Manzoor. Primary education for all: Learning from the BRAC experience: A case study, Dhaka, Abel Press, 1993.

Ahmed, Manzoor. The economics of non-formal education: Resources, costs and benefits, Nueva York, Praeger, 1975. 
Anderson, Stephen (Ed.). School improvement in the developing world: Case studies of the Aga Khan Foundation projects, Amsterdam, Swets and Zeitlinger, 2002.

Bauman, Kurt, J. "One million homeschooled students", en Teachers College Record, February 16, 2005. Disponible en www.tcrecord.org/Content. asp?ContentID=11756.

Benveniste, Patrick J., y McEwan, Luis. "The politics of rural school reform: Escuela Nueva in Colombia", en Journal of Education Policy, 16(6), 2001, pp. 547-599.

BRAC. Education programme. Disponible en www.brac.net/education. Fecha de acceso, 4 de abril de 2016.

Bransford, John D., Brown, Ann L., y Cocking, Rodney R. (Eds.). How people learn: Brain, mind, experience and schooling, Washington, DC, Commission on Behavioral and Social Sciences, National Research Council, National Academy Press, 2000.

Caillods, Françoise (Ed.). The prospects for educational planning, Paris, International Institute for Educational Planning, 1989.

Caine, Renate y Caine, Geoffrey. Education on the edge of possibility, Alexandria, VA, Association for Supervision and Curriculum Development, 1997.

City, Elizabeth A., Elmore, Richard F. y Lynch, Doug. "Redefining education: The future of learning is not the future of schooling", en Jal Mehta, Robert B. Schwartz, y Frederick M. Hess (Eds.), The futures of school reform, Cambridge, MA, Harvard Education Press, 2012, pp. 151-176.

Clandinin, Jean y Connelly, F. Michael. "Stories to live by: Narrative understandings of school reform", en Curriculum Inquiry, 28(2), 1998, pp. 149-164.

Colbert, Vicky y Jairo Arboleda. "Universalization of primary education in Colombia: The new school program", Ponencia presentada en la World Conference on Education for All, Jomtien, Tailandia, 1990.

Coombs, Philip. "Non-formal education: Myths, realities and opportunities", en Comparative Education Review, 20(3), 1976, pp. 281-293.

Coombs, Philip y Ahmed, Manzoor. Attacking rural poverty: Now nonformal education can help, Baltimore, Johns Hopkins University Press, 1974. 
Davies, Lynn. "The management and mismanagement of school effectiveness", en John D. Turner (Ed.), The state and the school: An international perspective, Washington, DC, Falmer Press, 1995, pp. 91-107.

DeStefano, Joseph, Hartwell, Ash, Schuh, Audrey-marie, y Benbow, Jane. Meeting EFA: Cost-effectiveness of complementary approaches, Washington, DC, Academy for Educational Development, 2005.

Elmore, Richard F. "Getting to scale with good educational practice”, en Harvard Educational Review, 66(1), 1996, pp. 1-26.

Evans, David. The planning of non-formal education, Paris, International Institute for Educational Planning, 1981.

Fagerlind, Ingemar y Sjostedt, Britt. Review and prospects of educational planning and management in Europe, París: UNESCO/International Congress on Planning and Management in Europe, 1990.

Farrell, Joseph, P. "Community education in developing countries: The quiet revolution in schooling", en F. Michael Connelly, Ming Fang He y Joanne Phillion (Eds.), Sage international handbook on curriculum and instruction, Thousand Oaks, CA, Sage Publications, 2008, pp. 369-390.

Farrell, Joseph P. "Alternative pedagogies and learning in alternative schooling systems in developing nations", Ponencia presentada en el congreso anual de la Comparative and International Education Society, Salt Lake City, Utah, 2004.

Farrell, Joseph, P. "Transformación de las formas de educación en el mundo en desarrollo. La aparición de un modelo de educación radicalmente alternativo: El papel transformador de la "Escuela Nueva" de Colombia", en Memorias del Primer Congreso Internacional de Escuelas Nuevas, Armenia, Colombia, Ministerio de Educación, 2003, pp. 71-103.

Farrell, Joseph P. "Why is educational reform so difficult? Similar descriptions, different prescriptions, failed explanations", en Curriculum Inquiry, 30(1), 2000, pp. 83-103.

Farrell, Joseph P. "Improving learning: Perspectives for primary education in rural Africa", Reporte comparado de contexto preparado para el seminario de mejora de la educación primaria en África, Lusaka, Zambia, UNESCO/Banco Mundial, 1998. 
Farrell, Joseph, P. "A retrospective on educational planning in comparative education", en Comparative Education Review, 41(3), 1997, pp. 277-313.

Farrell, Joseph P. "International lessons for school effectiveness: The view from the Third World", en Mark Holmes, Kenneth Leithwood y Donald Mussela (Eds.), Policy for effective schools, Nueva York, Teachers College Press y OISE, 1989, pp. 131-153.

Fullan, Michael, y Langworthy, Maria. A rich seam: How new pedagogies find deep learning, Londres, Pearson, 2014.

Fullan, Michael, y Quinn, Joanne. Coherence: The Right Drivers in Action for Schools, Districts, and Systems, Thousand Oaks, CA, Corwin, 2015.

Fullan, Michael, y Rincón-Gallardo, Santiago. "Developing High Quality Public Education in Canada: The Case of Ontario", en Frank Adamson, Björn Astrand, y Linda Darling-Hammond (Eds.), Global Education Reform: How privatization and public investment influence education outcomes, Nueva York, Routledge, 2016, pp. 169-193.

Fullan, Michael, y Watson, Nancy. "School-based management: Reconceptualizing to improve learning outcomes", Ponencia preparada para el seminario de mejora de resultados de aprendizaje en el Caribe del Banco Mundial, 1999.

Gloeckner, Gene W. y Jones, Paul. "Reflections on a decade of changes in homeschooling and the homeschooled into higher education", en Peabody Journal of Education, 88(8), 2013, pp. 309-323, doi: 10.1080/0161956X.2013.796837

Gouvernement du Québec, Ministère de l'Éducation. Québec education program: Elementary education, Quebec, Canadá, Ministère de l'Éducation, 2000.

Haiplik, Brenda. BRAC's non-formal education program (NFEP). Washington, DC, Academy for International Development, 2004.

Hargreaves, Andy, y Shirley, Dennis. The global fourth way: The quest for educational excellence, Thousand Oaks, CA, Corwin, 2012.

Martin-Chang, Sandra Lyn, Gould, Odette Noella y Meuse, Reanne E. "The impact of schooling on academic achievement: Evidence from homeschooled and traditionally schooled students", en Canadian Journal of Behavioural Science, 43(3), 2011, pp. 195-202. 
McGinn, Noel F. "Resistance to good ideas: Escuela Nueva in Colombia", en Lene Buchert (Ed.), Education reform in the South in the 1990s, París, UNESCO, 1998, pp. 29-52.

Melaville, Atelia, Berg, Amy C., y Blank, Martin J. Community based learning: Engaging students for success and citizenship, Washington, DC, Coalition for Community Schools, 2006.

Mourshed, Mona, Chijioke, Chinezi y Barber, Michael. How the world's most improved school systems keep getting better. Londres, McKinsey and Company, 2010.

Olson, David. Psychological theory and educational reform: How school remakes mind and society, Cambridge, Cambridge University Press, 2003.

Pitt, Jennifer. Case study for Escuela Nueva program, Washington, DC, Academy for Educational Development, 2004.

Pitt, Jennifer. Civic education and citizenship in Escuela Nueva schools in Colombia (Tesis de maestría sin publicar), OISE/University of Toronto, 2002.

Polyzoi, Eleoussa, Fullan, Michael y Anchan, John P. Change forces in post-communist Eastern Europe: Education in transition, Londres, Routledge/Falmer, 2003.

Psacharopoulos, George, Rojas, Carlos y Velez, Eduardo. "Achievement evaluation of Colombia's Escuela Nueva. Is multigrade the answer?", en Policy Research Working, Paper 896, Washington, DC, World Bank, 1992.

Ravitch, Diane y Vinovskis, Maris (Eds.). Learning from the past: What history teaches us about school reform, Baltimore, Johns Hopkins University Press, 1995.

Ray, Brian D. "Homeschooling associated with beneficial learner and societal outcomes but educators do not promote it", en Peabody Journal of Education, 88(3), 2013, pp. 324341. doi:10.1080/0161956X.2013.798508

Reimers, Fernando (Ed.). Unequal schools, unequal chances: The challenges to equal opportunity in the Americas, Cambridge, MA, Harvard University Press, 2000.

Rincón-Gallardo, Santiago. "Large-Scale Pedagogical Transformation as Widespread Cultural Change in Mexican Public Schools", en Journal of Educational Change, 17(4), 2016, pp. 411-436. 
Rogers, Alan. Non-formal education: Flexible schooling or participatory education?, Hong Kong, Comparative Education Research Centre, Universidad de Hong Kong, 2004.

Rodríguez, Encarna. Pedagogies and curriculums to (re)Imagine public education: Transnational tales of hope and resistance, Singapur, Springer, 2015.

Sahlberg, P. Finnish Lessons: What can the world learn from educational change in Finland?, Nueva York, Teachers College Press, 2011.

Schiefelbein, Ernesto. "School performance problems in Latin America: The potential role of the Escuela Nueva system", Ponencia preparada para el 2o Congreso International de Escuelas Nuevas. Medellín, Colombia, 2006.

Schiefelbein, Ernesto. In search of the school of the 21st century: Is Colombia's Escuela Nueva the right pathfinder?, Santiago, Chile, UNESCO, Oficina Regional para América Latina y el Caribe, 1993.

Scott, Suzanne. Education for child garment workers in Bangladesh (Tesis de maestría sin publicar). OISE/University of Toronto, 1996.

Shirley, Dennis. The New Imperatives of Educational Change: Achievement with Integrity, Nueva York y Londres, Routledge, 2017.

Siabato, Ricardo. Educación básica primaria en zonas rurales: La Escuela Nueva y su relación con el plan de universalización de la educación básica primaria, Bogotá, Ministerio de Educación, 1997.

Sweetser, Anne T. Lessons from the BRAC non-formal primary education program, Washington, DC, Academy for Educational Development, 1999.

Tyack, David, y Cuban, Larry. Tinkering toward utopia: A century of public school reform, Cambridge, MA, Harvard University Press, 1995.

Zavadsky, Heather. Bringing school reform to scale: Five awardwinning urban districts, Cambridge, MA, Harvard Education Press, 2009. 